\title{
Lyapunov Characterization for the Stability of Stochastic Control Systems
}

\author{
Fakhreddin Abedi, ${ }^{1,2}$ Wah June Leong, ${ }^{1}$ and Mohammad Abedi ${ }^{3}$ \\ ${ }^{1}$ Department of Mathematics, Universiti Putra Malaysia, 43400 Serdang, Selangor, Malaysia \\ ${ }^{2}$ Department of Mathematics, Institute for Advanced Studies in Basic Sciences (IASBS), Zanjan 45137 66731, Iran \\ ${ }^{3}$ Faculty of Mechanical and Energy Engineering, Shahid Beheshti University, Tehran 16589 53571, Iran
}

Correspondence should be addressed to Fakhreddin Abedi; f_abedi1352@yahoo.com

Received 20 May 2014; Accepted 21 August 2014

Academic Editor: Giuseppe Rega

Copyright (C) 2015 Fakhreddin Abedi et al. This is an open access article distributed under the Creative Commons Attribution License, which permits unrestricted use, distribution, and reproduction in any medium, provided the original work is properly cited.

Lyapunov-like characterization for the problem of input-to-state stability in the probability of nonautonomous stochastic control systems is established. We extend the well-known Artstein-Sontag theorem to derive the necessary and sufficient conditions for the input-to-state stabilization of stochastic control systems. Illustrating example is provided.

\section{Introduction}

The stabilization of various types of nonlinear systems has been widely studied in the past years, see, for instance, [15]. The necessary and sufficient conditions for input-to-state stability and robust stability at the equilibrium state of nonlinear system are provided by Sontag and Wang [1]. Angeli et al. [2] showed that there exists a positive definite Lyapunov function whose derivative along the system is a negative definite that guarantees a time-varying system satisfying in the integral input-to-state stability property. Later, Grune [3] derived a suitable Lyapunov function and established an input-to-state dynamical stability property for a timevarying system. The necessary and sufficient conditions for input-to-state stability of nonlinear time-varying system have been provided by Karafyllis and Tsinias [4]. Ning et al. [5] employed an indefinite Lyapunov function rather than a negative definite function and established input-to-state stability and integral input-to-state stability of time-varying system.

Tsinias [6], Florchinger [7], Krstic and Deng [8], Deng et al. [9], van Handel [10], and Abedi et al. [11-13] attempted in several directions to cover global asymptotic stability in probability (GASP) and input-to-state stability in probability (ISSP) of stochastic differential systems (SDS) by Lyapunov functions.

The main purpose of this paper is to establish a Lyapunov characterization for the problem of ISSP of nonautonomous stochastic control systems (NSCS). We extend the wellknown Artstein-Sontag theorem (see $[14,15]$ ) established in Karafyllis and Tsinias [4] to the concept of stochastic control Lyapunov function (CLF) in order to derive the necessary and sufficient conditions for the ISSP of NSCS. We also establish the existence of an explicit formula of a feedback law exhibiting ISSP property and give some applications to feedback stabilization. The analysis used in this paper is closely related to that of Karafyllis and Tsinias [4].

The paper is organized as follows. In Section 2, we introduce the class of stochastic systems and some basic definitions and results that we are dealing with in this paper. We also describe a wider class of NSCS and we focus on the properties of stochastic CLF which play an important role in ISSP property. Finally, in Section 3, we state and prove the main results of the paper on the ISSP property of NSCS. We also provide a numerical example to illustrate our results. 


\section{Fundamental Definitions and Results}

The purpose of this section is to introduce the notion of robust stability in probability (RSP) and ISSP property for a class of stochastic systems. For a detailed presentation of stochastic stability theory, we refer the reader to the book of Khasminskii [16] and the paper of Abedi et al. [11].

Let $(\Omega, F, P)$ be a complete probability space and, for any $k \in\{1, \ldots, m\}$, denote by $\left(w_{t}^{k}\right)_{t \geq 0}$ a standard $R^{m}$-valued Wiener process defined on this space.

We consider the SDS

$$
\begin{array}{r}
d x=f(t, x, v) d t+\sum_{k=1}^{m} h_{k}(t, x, v) d w_{t}^{k} \\
x \in R^{n}, \quad v \in R^{l}, \quad t \geq 0,
\end{array}
$$

where

(i) the functions $f: R^{+} \times R^{n} \times R^{l} \rightarrow R^{n}, h_{k}: R^{+} \times R^{n} \times$ $R^{l} \rightarrow R^{n \times m}$, and $1 \leq k \leq m$ are locally Lipschitz with respect to $(x, v)$ with $f(t, 0, v)=0$ and $h_{k}(t, 0, v)=0$; in the sense that for every bounded interval $I \subset R^{+}$ and for every compact subset $S$ of $R^{n} \times R^{l}$, there exists a constant $C \geq 0$ such that

$$
\begin{gathered}
|f(t, x, v)-f(t, y, \widetilde{v})|+\sum_{k=1}^{m}\left|h_{k}(t, x, v)-h_{k}(t, y, \widetilde{v})\right| \\
\leq C(|x-y|+|v-\widetilde{v}|) . \\
\forall t \in I, \quad(x, v),(y, \widetilde{v}) \in S,
\end{gathered}
$$

where, throughout this paper, $|\cdot|$ denotes the usual Euclidean norm.

(ii) $x_{t}=x(t)=\left(t, t_{0}, x_{0}, v\right)$ is a solution of (1) at time $t$ that corresponds to some input $v \in L_{\mathrm{loc}}^{\infty}$, initiated from $x_{0}$ at time $t_{0}$.

Definition 1. A function $\gamma: R^{+} \rightarrow R^{+}$is

(i) a $K$-function if it is continuous, strictly increasing and $\gamma(0)=0$

(ii) a $K_{\infty}$-function if it is a $K$-function and also $\gamma(r) \rightarrow$ $\infty$ as $r \rightarrow \infty$,

(iii) a positive definite function if $\gamma(r)>0$ for all $r>0$, and $\gamma(0)=0$, and

(iv) a $K^{+}$-function if it is a positive nondecreasing $C^{\infty}$ function.

Definition 2. The equilibrium $x_{t} \equiv 0$ of the system (1) is

(i) globally stable in probability, if, for every $\epsilon>0$ and input $v(t)$, there exists a class $K$-function $\gamma(\cdot)$ such that

$$
P\left\{|x(t)|<\gamma\left(\left|x_{0}\right|\right)\right\} \geq 1-\epsilon, \quad \forall t \geq 0, \forall x_{0} \in R^{n} \backslash\{0\},
$$

(ii) globally asymptotically stable in probability, if it is globally stable in probability and

$$
P\left\{\lim _{t \rightarrow \infty}|x(t)|=0\right\}=1, \quad \forall x_{0} \in R^{n} .
$$

In the following, we recall the stochastic version of La Salle's invariant theorem established by Kushner [17].

Theorem 3. Suppose that there exists a Lyapunov function $\Phi$ defined on $R^{+} \times R^{n}$ such that

$$
\mathbf{D} \Phi(t, x) \leq 0,
$$

where $\mathbf{D}$ is the infinitesimal generator of the stochastic process solution of stochastic system (1) as follows:

$$
\begin{aligned}
\mathrm{D} \Phi(t, x)= & \frac{\partial \Phi(t, x)}{\partial t}+\sum_{i=1}^{n} f^{i} \frac{\partial \Phi(t, x)}{\partial x_{i}} \\
& +\frac{1}{2} \sum_{i, j=1}^{n} \sum_{k=1}^{m} h_{k}^{i} h_{k}^{j} \frac{\partial^{2} \Phi(t, x)}{\partial x_{i} \partial x_{j}} .
\end{aligned}
$$

Then, the equilibrium solution $x_{t} \equiv 0$ of stochastic system (1) tends to the largest invariant set whose support is contained in the locus $\mathrm{D} \Phi\left(x_{t}\right)=0$ for any $t \geq 0$ with probability 1 .

We will now turn the attention to a wider class of NSCS and focus on the properties of stochastic CLF which play an important role in ISSP property in Section 3.

Denote by $x(t) \in R^{n}$ the stochastic process solution of the NSCS written in the sense of Ito:

$$
\begin{aligned}
& d x=f(t, x, v) d t+\sum_{z=1}^{p} g_{z}(t, x) u^{z} d t \\
& \quad+\sum_{k=1}^{m} h_{k}(t, x, v) d w_{t}^{k} \\
& x \in R^{n}, \quad v \in R^{l}, \quad u \in R^{p}, \quad t \geq 0,
\end{aligned}
$$

where the dynamics $f(\cdot), h_{k}(\cdot)$, and $g_{z}: R^{+} \times R^{n} \rightarrow R^{n \times p}$, $1 \leq z \leq p$ are $C^{0}$ and locally Lipschitz with respect to $(x, v)$ with $f(t, 0, v)=0$ and $h_{k}(t, 0, v)=0$.

We recall the definition of RSP introduced by Abedi and Leong [18] on the neighborhood $D \subset D^{n}$ of the origin as follows.

Definition 4. The NSCS (7) is said to be RSP if there exists a neighborhood $D$ of the origin in $R^{n}$ and a function $k: R^{+} \times$ $D \rightarrow R^{n}$, with $k(t, 0)=0$, such that

(i) for every $x$ remains in $D$, for all $t \geq 0$, the solution $x_{t}$ of the resulting closed-loop system

$$
\begin{aligned}
d x= & f(t, x, v) d t+\sum_{z=1}^{p} g_{z}(t, x) k(t, x)^{z} d t \\
& +\sum_{k=1}^{m} h_{k}(t, x, v) d w_{t}^{k}
\end{aligned}
$$

where $\left(w_{t}\right)_{t \geq 0}$ is a standard $R^{m}$-valued Wiener process defined on a complete probability space $(\Omega, F, P)$, is uniquely defined with $v$ as input and

(ii) the equilibrium solution $x_{t} \equiv 0$ of the resulting closed-loop system (8) is GASP with $v$ as input. 
Consider the NSCS (7), under a slight change of hypothesis in the notion of ISS property introduced by Karafyllis and Tsinias [4], we obtain the notion of ISSP in terms of Lyapunov functions as follows.

Definition 5. Let $\gamma(t, x): R^{+} \times R^{+} \rightarrow R^{+}$be a positive definite function, which is $C^{0}$, locally Lipschitz in $x$; the NSCS (7) satisfies the weak ISSP (wISSP) from the input $v$ with gain $\gamma(\cdot)$ if each solution of NSCS (7) exists for all $t \geq t_{0}$ and satisfies conditions (3) and (4) of Definition 2 provided that

$$
|v(\cdot)| \leq \gamma(t,|x|) .
$$

Definition 6. We say that NSCS (7) satisfies the ISSP from the input $v$ with gain $\gamma(\cdot)$ if the following conditions hold:

(i) the NSCS (7) satisfies wISSP property and

(ii) the map $\gamma(\cdot)$ is of class $K_{\infty}$ function for each $t \geq 0$.

As in the deterministic case (see $[4,19]$ ), we can easily establish the following elementary result.

Lemma 7. The NSCS (7) satisfies the wISSP property if and only if $0 \in R^{n}$ is $R S P$ for the system

$$
\begin{gathered}
d x=f(t, x, \gamma(t,|x|) d) d t+\sum_{z=1}^{p} g_{z}(t, x) k(t, x)^{z} d t \\
+\sum_{k=1}^{m} h_{k}(t, x, \gamma(t,|x|) d) d w_{s}^{k} \\
\quad x \in R^{n}, \quad d \in B[0,1] \subset R^{q}, \quad t \geq 0 .
\end{gathered}
$$

Proof. The proof of this lemma is a direct consequence of Definitions 2, 4, and 5 and the fact that each solution $x(t)$ of the NSCS (10) that corresponds to some $v(\cdot)$ coincides with the solution of the NSCS (7) with the same initial $x_{0}$ and $t_{0}$ and corresponding to $v=\gamma(t,|x|) d$, namely, satisfying (9). Conversely, each solution $x(t)$ of the NSCS (7) under restriction (9) is a solution of the NSCS (10) with input $d=$ $v / \gamma(t,|x|)$ and the same initial value.

The main results of this paper (Theorems 11 and 12) constitute the extensions of the well-known Artstein-Sontag theorem established in Karafyllis and Tsinias [4] and guarantee the existence of a $C^{\infty}$ mapping $u=k(t, x)$ in such a way that the resulting closed-loop system (8) satisfies ISSP property with $v$ as input.

We denote by $\mathbf{D}$ the infinitesimal generator of the stochastic process solution of NSCS (7); that is, D is the second-order differential operator defined for any function $\Phi$ in $C^{1,2}\left(R^{+} \times\right.$ $\left.R^{n}, R\right)$ by

$$
\begin{aligned}
\mathbf{D} \Phi(t, x)= & \frac{\partial \Phi(t, x)}{\partial t}+\sum_{i=1}^{n} f^{i} \frac{\partial \Phi(t, x)}{\partial x_{i}} \\
& +\frac{1}{2} \sum_{i, j=1}^{n} \sum_{k=1}^{m} h_{k}^{i} h_{k}^{j} \frac{\partial^{2} \Phi(t, x)}{\partial x_{i} \partial x_{j}}
\end{aligned}
$$

where $1 \leq i$ and $j \leq n$. We also denote by $\mathbf{D}_{z}, 1 \leq z \leq p$ the first-order differential operator defined for any function $\Phi$ in $C^{1}\left(R^{+} \times R^{n}, R\right)$ by

$$
\mathbf{D}_{z} \Phi(t, x)=\sum_{i=1}^{n} g_{i}^{z}(t, x) \frac{\partial \Phi(t, x)}{\partial x_{i}}
$$

The following definition is an extension of Definition 2.4 established in [11] and which described the stochastic CLF that was used for ISSP of NSCS (7) at the origin.

Definition 8. Let $\gamma(t, x): R^{+} \times R^{+} \rightarrow R^{+}$be a positive definite function, which is $C^{0}$, locally Lipschitz in $x$, the NSCS (7) admits a stochastic CLF, if there exists a $C^{1,2}$ function $\Phi: R^{+} \times R^{n} \rightarrow R^{+}$, class $K_{\infty}$ functions $a_{1}, a_{2}$, and a positive definite function $\rho: R^{+} \rightarrow R^{+}$such that, for all $(t, x, d) \in R^{+} \times R^{n} \times L$, the following conditions hold:

$$
\begin{gathered}
a_{1}(t,|x|) \leq \Phi(t, x) \leq a_{2}(t,|x|) \\
\mathbf{D}_{z} \Phi(t, x)=0, \quad|v| \leq \gamma(t,|x|) \\
\Longrightarrow \mathbf{D} \Phi(t, x) \leq-\rho(\Phi(t, x)) .
\end{gathered}
$$

Since the differential operator $\mathbf{D}$ appears in the condition (14) stated in Definition 8, the computations in the stochastic case are more tedious than those in the deterministic case introduced by Karafyllis and Tsinias [4]. We extended the concept of CLF introduced by Karafyllis and Tsinias [4] and obtained the notion of stochastic CLF to Definition 8 that was used for ISSP of the NSCS (7) at the equilibrium state. Relationships between the concepts of ISSP given above and their characterization in terms of Lyapunov functions are developed in the next section. In order to establish our main results as in Theorem 11, we need the following technical theorem established in [18]. The proof of this theorem was exposed in [18] and is therefore omitted.

Theorem 9. Consider the NSCS (7). Then the following statements are equivalent:

(i) there exists a $C^{\infty}$ function $k: R^{+} \times R^{n} \rightarrow R^{p}$ with $k(t, 0)=0$ in such a way that the resulting closed-loop system (8) satisfies RSP property;

(ii) there exists a $C^{0}$ function $k: R^{+} \times R^{n} \rightarrow R^{p}$ with $k(t$, $0)=0$ being locally Lipschitz in $x$, in such a way that the resulting closed-loop system (8) satisfies RSP property;

(iii) the NSCS (7) admits a stochastic CLF.

\section{Main Results}

We study the problem of finding continuous feedback law in order to derive necessary and sufficient conditions for ISSP of NSCS. For the NSCS (7), we extend the well-known ArtsteinSontag theorem established in [4] by introducing the concept of stochastic CLF (Theorem 11). This result is a useful tool for designing an explicit formula of a feedback law that exhibits 
ISSP of the resulting closed-loop system (8) at the equilibrium state (Theorem 12).

Proposition 10. Consider the system (7) and let $\gamma(t, x): R^{+} \times$ $R^{+} \rightarrow R^{+}$be a positive definite function, which is $C^{0}$, locally Lipschitz in $x$, and further assume that NSCS (8) satisfies the wISSP property from the input $v$ with gain $\gamma(\cdot)$. Then, the NSCS (7) admits a stochastic CLF.

Proof. The proof of this proposition is a direct consequence of Theorem 9 and Lemma 7. Indeed, assume that the resulting closed-loop system (8) satisfies the wISSP property; then, according to Lemma 7, the resulting closed-loop system (8) satisfies the RSP property. Therefore, by Theorem 9, the NSCS (7) admits a stochastic CLF.

The input-to-state stability results proven in this paper use a technique which is a combination of Karafyllis and Tsinias [4] decomposition in deterministic case and Abedi et al. [11] decomposition in stochastic case. We use this decomposition and extend the existing input-to-state stability results. The following theorem is an extension of the wellknown Artstein-Sontag theorem established in [4] (Theorem 5.1 of [4]). The proof of this theorem is a stochastic analogue of the deterministic proof of Karafyllis and Tsinias [4]. In the proof of this theorem, we use the stochastic versions of Artstein-Sontag theorem established in Abedi et al. [11] and La Salle's invariance theorem developed in [17].

Theorem 11. Consider the NSCS (7) and let $\gamma(t, x): R^{+} \times$ $R^{+} \rightarrow R^{+}$be a positive definite function, which is $C^{0}$, locally Lipschitz in $x$. Then the following statements are equivalent:

(i) there exists a $C^{\infty}$ function $k: R^{+} \times R^{n} \rightarrow R^{p}$ with $k(t, 0)=0$ such that the resulting closed-loop system (8) satisfies the wISSP property with gain $\gamma(\cdot)$ from the input $v$. It turns out that the resulting closed-loop system (8) satisfies the ISSP property;

(ii) there exists a $C^{0}$ function $k: R^{+} \times R^{n} \rightarrow R^{p}$ with $k(t, 0)=0$ for all $t \geq 0$, being locally Lipschitz in $x$, such that the resulting closed-loop system (8) satisfies the same property as that in statement (i);

(iii) the NSCS (7) admits a stochastic CLF.

Proof. (i $\rightarrow \mathrm{ii}$ ) is obvious.

(ii $\rightarrow$ iii) Suppose that there exists a function $k(t, x)$, such that the closed-loop system (8) satisfies the wISSP with gain $\gamma(\cdot)$ from the input $v$. According to Lemma 7, the resulting closed-loop system (8) satisfies the RSP property. Then, by Proposition 10 and the converse Lyapunov theorem (Theorem 2) established by Kushner [20], which provided the existence of a Lyapunov function in some neighborhoods of the origin, there exists a $C^{1,2}$ function $\Phi: R^{+} \times R^{n} \rightarrow R^{+}$and a continuous and positive definite function $\phi(t, x)$ such that (13) holds and

$$
\begin{aligned}
|v| \leq \gamma(t,|x|) & \\
\Longrightarrow \mathbf{D}_{0} \Phi(t, x) & =\mathbf{D} \Phi(t, x)+\sum_{z=1}^{p} \mathbf{D}_{z} \Phi(t, x) k(t, x)^{z} \\
& \leq-\phi(t, x),
\end{aligned}
$$

where $\mathbf{D}_{0}$ is the infinitesimal generator of the resulting closed-loop system (8). The latter inequality implies (14) where $\rho(\Phi(t, x))=\phi(t, x)$ and the condition $\mathbf{D}_{z} \Phi(t, x)=0$. Therefore, the requirement in Definition 8 holds and $\Phi(t, x)$ is a stochastic CLF for the NSCS (7).

(iii $\rightarrow$ i) Assume that the NSCS (7) admits a stochastic CLF. Consider the functions $a_{1}, a_{2}$, and $\Phi$ as defined in (13) and (14). From (13) we have

$$
\frac{\partial \Phi}{\partial t}(t, 0)=0, \quad \frac{\partial \Phi}{\partial x}(t, 0)=0 .
$$

Condition (14) in conjunction with (16) enables us to build, by standard partition of unity arguments, a $C^{\infty}$ map $k: R^{+} \times$ $R^{n} \rightarrow R^{p}$ with $k(t, 0)=0$ such that

$$
\begin{aligned}
\mathbf{D}_{0} \Phi & (t, x) \\
& =\max _{|v| \leq \gamma(t,|x|)} \mathbf{D} \Phi(t, x)+\sum_{z=1}^{p} \mathbf{D}_{z} \Phi(t, x) k(t, x)^{z} \\
& \leq-\rho(\Phi(t, x)) .
\end{aligned}
$$

From (17) and $\Phi(t, x) \geq 0, \Phi_{t}=\Phi(t, x)$ is a supermartingale. By a supermartingale inequality established by Rogers and Williams [21], for any class $K_{\infty}$ function $\Gamma(\cdot)$, we have

$$
P\left\{\sup _{0 \leq \tau \leq t} \Phi_{\tau} \geq \Gamma\left(\Phi_{t}\right)\right\} \leq \frac{E\left(\Phi_{t}\right)}{\Gamma\left(\Phi_{t}\right)} .
$$

Let $\Phi_{0}=\Phi\left(0, x_{0}\right)$. By using Ito formula for NSCS (7), we obtain

$$
\begin{aligned}
\Phi_{t}= & \Phi_{0}+\int_{0}^{t}\left(\mathbf{D} \Phi(s, x)+\sum_{z=1}^{p} \mathbf{D}_{z} \Phi(s, x) k(s, x)^{z}\right) d s \\
& +\int_{0}^{t} \sum_{k=1}^{m} h_{k}(s, x, v) \frac{\partial \Phi}{\partial x}(s, x) d w_{s} .
\end{aligned}
$$

By taking into account (17) and (19) we get

$$
\begin{aligned}
\Phi_{t}= & \Phi_{0}+\int_{0}^{t} \mathbf{D}_{0} \Phi(s, x) d s \\
& +\int_{0}^{t} \sum_{k=1}^{m} h_{k}(s, x, v) \frac{\partial \Phi}{\partial x}(s, x) d w_{s} .
\end{aligned}
$$

From (20) we have

$$
E\left[\Phi_{t}\right]=\Phi_{0}+E\left(\int_{0}^{t} \mathbf{D}_{0} \Phi(s, x) d s\right) .
$$


Invoking (17) and (21) yields

$$
E\left[\Phi_{t}\right] \leq \Phi_{0}+E\left(\int_{0}^{t}-\rho(\Phi(s, x)) d s\right) \leq \Phi_{0} .
$$

Hence, from (18) and (22), we have

$$
P\left\{\sup _{0 \leq \tau \leq t} \Phi_{\tau} \geq \Gamma\left(\Phi_{t}\right)\right\} \leq \frac{\Phi_{0}}{\Gamma\left(\Phi_{t}\right)} .
$$

Thus,

$$
P\left\{\sup _{0 \leq \tau \leq t} \Phi_{\tau}<\Gamma\left(\Phi_{t}\right)\right\} \geq 1-\frac{\Phi_{0}}{\Gamma\left(\Phi_{t}\right)} .
$$

For a given $K_{\infty}$ functions $a_{1}, a_{2}$, and $\Gamma$, define $\beta=a_{1}^{-1}$ 。 $\Gamma \circ a_{2}$. Then $\sup _{0 \leq \tau \leq t} \Phi_{\tau}<\Gamma\left(\Phi_{t}\right)$ implies that

$$
\sup _{0 \leq \tau \leq t}|x(t)|<\beta\left(\left|x_{0}\right|\right)
$$

and so,

$$
P\left\{\sup _{0 \leq \tau \leq t}|x(t)|<\beta\left(\left|x_{0}\right|\right)\right\} \geq 1-\frac{\Phi_{0}}{\Gamma\left(\Phi_{t}\right)} .
$$

For a given $\epsilon>0$, choose $\Gamma\left(\Phi_{t}\right)$ such that

$$
\Gamma\left(\Phi_{t}\right) \geq \frac{\Phi_{0}}{\epsilon} .
$$

By taking into account (26) and (27), we obtain

$$
P\left\{\sup _{0 \leq \tau \leq t}|x(t)|<\beta\left(\left|x_{0}\right|\right)\right\} \geq 1-\epsilon .
$$

The latter inequality implies that

$$
\begin{array}{r}
P\left\{|x(t)|<\beta\left(\left|x_{0}\right|\right)\right\} \geq 1-\epsilon, \\
\forall t \geq 0, \quad \forall x_{0} \in R^{n} \backslash\{0\} .
\end{array}
$$

Thus, from (28) and Definition 4, we have that the equilibrium is globally stable in probability with respect to the resulting closed-loop system (8). On the other hand, from (17) and the stochastic version of La Salle's invariance Theorem 3, we obtain the stochastic process solution $x(t)$ of the resulting closed-loop system (8), which tends to 0 with probability 1 ; that is,

$$
P\left\{\lim _{t \rightarrow \infty}|x(t)|=0\right\}=1
$$

Therefore, from (30) and the above global stability in probability and Definition 4, we get the equilibrium is RSP with respect to the resulting closed-loop system (8). The desired wISSP property for the resulting closed-loop system (8) is a consequence of Lemma 7.

The following theorem, which is an immediate consequence of Theorem 11, is a stochastic extension of Proposition 5.2 in [4]. The proof of this theorem is a stochastic analogue of the deterministic proof of Karafyllis and Tsinias [4]. In the proof of this theorem, we use an explicit formula of a feedback law derived by Florchinger and Verriest [7] in exhibiting wISSP property for the resulting closed-loop system (8).

Theorem 12. Let $\gamma(t, x): R^{+} \times R^{+} \rightarrow R^{+}$be a positive definite function, which is $C^{0}$, locally Lipschitz in $x$, and further assume $\Phi$ is a stochastic CLF associated with the NSCS (7), and, for any $(t, x) \in R^{+} \times R^{n}$, denote by $b(t, x)$ and $\Psi(t, x)$ the functions defined by

$$
\begin{gathered}
b(t, x)=\mathbf{D}_{z} \Phi(t, x), \\
\Psi(t, x)=\max _{|v| \leq \gamma(t,|x|)} \mathbf{D} \Phi(t, x)+\rho(\Phi(t, x)),
\end{gathered}
$$

then the feedback law

$$
k(t, x)=\xi\left(\Psi(t, x),(b(t, x))^{2}\right)(b(t, x)),
$$

where $b(t, x)$ and $\Psi(t, x)$ are given by (31) and (32), respectively, and

$$
\xi(\Psi, b)= \begin{cases}-\frac{\Psi+\sqrt{\Psi^{2}+b^{2}}}{b(1+\sqrt{1+b})} & \text { if } b>0, \\ 0 & \text { if } b=0,\end{cases}
$$

guarantees that the resulting closed-loop system (8) satisfies $w I S S P$ property with gain $\gamma(\cdot)$ from the input $v$.

Proof. Assume that the NSCS (7) admits a stochastic CLF. From (14) and (32), we have

$$
\mathbf{D}_{z} \Phi(t, x)=0 \Longrightarrow \Psi(t, x) \leq 0 \text {. }
$$

Notice that the feedback law

$$
k(t, x)= \begin{cases}-\frac{(\mathbf{D} \Phi(\cdot)+\rho(\Phi(\cdot)))+\sqrt{(\mathbf{D} \Phi(\cdot)+\rho(\Phi(\cdot)))^{2}+\left(\mathbf{D}_{z} \Phi(\cdot)\right)^{4}}}{\mathbf{D}_{z} \Phi(\cdot)\left(1+\sqrt{1+\left(\mathbf{D}_{z} \Phi(\cdot)\right)^{2}}\right)} & \text { if } \mathbf{D}_{z} \Phi(\cdot)>0, \\ 0 & \text { if } \mathbf{D}_{z} \Phi(\cdot)=0,\end{cases}
$$


is well defined for all $(t, x) \in R^{+} \times R^{n}$, since the denominator in (33) is strictly positive for all $(t, x) \in R^{+} \times R^{n}$ and is of class $C^{0}\left(R^{n}\right)$. Furthermore, according to regularity assumptions made for $\Phi(t, x), f(t, x, v), g_{z}(t, x), h_{k}(t, x, v), \gamma(t, x)$, and $\rho(t, x)$, the function $k(t, x)$ as defined by (33) is $C^{0}$ on $R^{+} \times R^{n}$ and locally Lipschitz with respect to $x \in R^{n}$, with $k(t, 0)=0$, for all $t \geq 0$.

Denoting by $\mathbf{D}_{0}$ the infinitesimal generator of the stochastic process solution of the resulting closed-loop system (8), we get

$$
\mathbf{D}_{0} \Phi(t, x)=\max _{|v| \leq \gamma(t,|x|)} \mathbf{D} \Phi(t, x)+\sum_{z=1}^{p} \mathbf{D}_{z} \Phi(t, x) k(t, x)^{z} .
$$

It then follows from (33) and (35) that

$$
\mathbf{D}_{0} \Phi(t, x) \leq-\rho(\Phi(t, x))
$$

From (38) and $\Phi(t, x) \geq 0$, we can conclude that $\Phi_{t}=$ $\Phi(t, x)$ is a supermartingale and, therefore, the rest of the proof is a straightforward consequence of (38) and Theorem 11 (implication (iii) $\rightarrow$ (i)). This completes the proof of Theorem 12. results.

Now, we can make the following summaries on our main

Remark 13. (i) The necessary and sufficient ISSP conditions for the NSCS (7) obtained in Theorem 11 (that is an extension of the well-known Artstein-Sontag theorem established in $[4])$ are different from those stated in $[1-5,19]$ in deterministic case and [6] in stochastic case.

(ii) We extended the concept of stochastic Lyapunov condition introduced in Definition 2.4 established in [11] and obtained the notion of stochastic CLF to Definition 8 that is used for ISSP of the NSCS (7) at the origin.

(iii) The stabilizability results proven for the stochastic systems in Abedi et al. [11] that established the necessary and sufficient conditions for global asymptotic stability of stochastic system and Tsinias [6] that obtained the sufficient conditions for global stability of triangular stochastic system do not permit us to establish the necessary and sufficient conditions for ISSP of the NSCS (7) at the origin, whereas the results of this paper are still valid. Furthermore, both the results and the proofs used in our paper, however, are different from those in $[6,11]$.

Finally, in this section, we illustrate our results by designing a numerical example.

Example 14. Denote by $x(t) \in R^{2}$ the solution of the SCS

$$
\begin{aligned}
d\left(\begin{array}{l}
x_{1} \\
x_{2}
\end{array}\right)= & \left(\begin{array}{c}
-x_{1}^{3}-x_{1} x_{2} \\
x_{1}-x_{2}+x_{1}^{2} \phi\left(x_{1}\right)
\end{array}\right) d t \\
& +\left(\begin{array}{c}
0 \\
x_{2}
\end{array}\right) u d t+\left(\begin{array}{c}
0 \\
x_{2}+\phi\left(x_{1}\right)
\end{array}\right) d w,
\end{aligned}
$$

where $w$ is a standard real-valued Wiener process, $u$ is a realvalued measurable control law, and $v=\phi\left(x_{1}\right)$ is a smooth functional mapping $R$ into $R$ such that

$$
\phi\left(x_{1}\right)<x_{1}^{2}
$$

for any $x_{1} \neq 0$. Obviously, the function $\Phi$, defined on $R^{n}$ by

$$
\Phi\left(x_{1}, x_{2}\right)=\frac{1}{2} x_{1}^{2}+\left(x_{2}+\phi\left(x_{1}\right)\right)^{4}
$$

is a stochastic CLF for SCS (39). Indeed, for any $x \neq 0$ with

$$
\begin{aligned}
\mathbf{D}_{z} \Phi(x) & =\sum_{i=1}^{n} g_{i}^{z}(x) \frac{\partial \Phi(x)}{\partial x_{i}} \\
& =g_{2} \frac{\partial \Phi(x)}{\partial x_{2}} \\
& =4 x_{2}\left(x_{2}+\phi\left(x_{1}\right)\right)^{3}=0,
\end{aligned}
$$

it follows that $x_{2}=-\phi\left(x_{1}\right)$, and therefore

$$
\begin{aligned}
\left.\mathbf{D} \Phi(x)\right|_{x_{2}=-\phi\left(x_{1}\right)} & =\sum_{i=1}^{n} f_{i} \frac{\partial \Phi(x)}{\partial x_{i}}+\frac{1}{2} \sum_{i, j=1}^{n} \sum_{k=1}^{m} h_{k}^{i} h_{k}^{j} \frac{\partial^{2} \Phi(x)}{\partial x_{i} \partial x_{j}} \\
& =-x_{1}^{2}\left(x_{1}^{2}-\phi\left(x_{1}\right)\right) .
\end{aligned}
$$

With condition $v=\phi\left(x_{1}\right)<x_{1}^{2}$, the latter equality implies $\mathrm{D} \Phi(x)<0$. Thus, by Definition 8 , the function $\Phi$ is a stochastic CLF for SCS (39). Therefore, according to Theorem 11, there exists a $C^{\infty}$-feedback law $k(x)$ with $k(0)=0$ such that wISSP property is fulfilled for the resulting closed-loop system deduced from SCS (39).

\section{Conclusions}

Lyapunov characterization for the problem of ISSP of stochastic control systems is given. Furthermore, we have extended the well-known Artstein-Sontag theorem established by Karafyllis and Tsinias [4] to the concept of stochastic CLF in deriving the necessary and sufficient conditions for ISSP property of stochastic control systems. We have also established the existence of an explicit formula of a feedback law exhibiting ISSP and given some applications to feedback stabilization.

\section{Conflict of Interests}

The authors declare that there is no conflict of interests regarding the publication of this paper.

\section{References}

[1] E. D. Sontag and Y. Wang, "Lyapunov characterizations of input to output stability," SIAM Journal on Control and Optimization, vol. 39, no. 1, pp. 351-359, 2000. 
[2] D. Angeli, E. D. Sontag, and Y. Wang, "A characterization of integral input-to-state stability," IEEE Transactions on Automatic Control, vol. 45, no. 6, pp. 1082-1096, 2000.

[3] L. Grune, "Input-to-state dynamical stability and its Lyapunov function characterization," IEEE Transactions on Automatic Control, vol. 47, no. 9, pp. 1499-1504, 2002.

[4] I. Karafyllis and J. Tsinias, "A converse Lyapunov theorem for nonuniform in time global asymptotic stability and its application to feedback stabilization," SIAM Journal on Control and Optimization, vol. 42, no. 3, pp. 936-965, 2003.

[5] C. Ning, Y. He, M. Wu, Q. Liu, and J. She, "Input-to-state stability of nonlinear systems based on an indefinite Lyapunov function," Systems \& Control Letters, vol. 61, no. 12, pp. 12541259, 2012.

[6] J. Tsinias, "Stochastic input-to-state stability and applications to global feedback stabilization," International Journal of Control, vol. 71, no. 5, pp. 907-931, 1998.

[7] P. Florchinger and E. I. Verriest, "Application of stochastic Artstein's theorem to feedback stabilization," Stochastic Analysis and Applications, vol. 18, no. 3, pp. 361-373, 2000.

[8] M. Krstic and H. Deng, Stabilization of Uncertain Nonlinear Systems, Springer, New York, NY, USA, 1998.

[9] H. Deng, M. Krstic, and R. J. Williams, "Stabilization of stochastic nonlinear systems driven by noise of unknown covariance," IEEE Transactions on Automatic Control, vol. 46, no. 8, pp. 12371253, 2001.

[10] R. van Handel, "Almost global stochastic stability," SIAM Journal on Control and Optimization, vol. 45, no. 4, pp. 1297-1313, 2006.

[11] F. Abedi, M. A. Hassan, and N. M. Arifin, "Lyapunov function for nonuniform in time global asymptotic stability in probability with application to feedback stabilization," Acta Applicandae Mathematicae, vol. 116, no. 1, pp. 107-117, 2011.

[12] F. Abedi, W. J. Leong, and S. S. Chaharborj, "Exponential inputto-state stability of composite stochastic systems," Advances in Difference Equations, vol. 2013, article 208, 10 pages, 2013.

[13] F. Abedi, W. J. Leong, and S. S. Chaharborj, "On the asymptotical and practical stability of stochastic control systems," Mathematical Problems in Engineering, vol. 2013, Article ID 560647, 10 pages, 2013.

[14] Z. Artstein, "Stabilization with relaxed controls," Nonlinear Analysis: Theory, Methods \& Applications, vol. 7, no. 11, pp. 11631173, 1983.

[15] E. D. Sontag, "A “universal" construction of Artstein's theorem on nonlinear stabilization," Systems \& Control Letters, vol. 13, no. 2, pp. 117-123, 1989.

[16] R. Z. Khasminskii, Stochastic Stability of Differential Equation, Sijthoff Noordhoff, Alphen aan den Rijn, The Netherlands, 1980.

[17] H. J. Kushner, "Stochastic stability," in Stability of Stochastic Dynamical Systems, vol. 294 of Lecture Notes in Mathematics, pp. 97-124, Springer, Berlin, Germany, 1972.

[18] F. Abedi and W. J. Leong, "Dynamic robust stabilization of stochastic differential control systems," IMA Journal of Mathematical Control and Information, vol. 30, no. 4, pp. 559-569, 2013.

[19] E. D. Sontag and Y. Wang, "On characterizations of the inputto-state stability property," Systems \& Control Letters, vol. 24, no. 5, pp. 351-359, 1995.

[20] H. J. Kushner, "Converse theorems for stochastic Liapunov functions," SIAM Journal on Control and Optimization, vol. 5, pp. 228-233, 1967.

[21] L. C. G. Rogers and D. Williams, Diffusions, Markov Processes and Martingales, vol. 1, Wiley, New York, NY, USA, 2nd edition, 1994. 


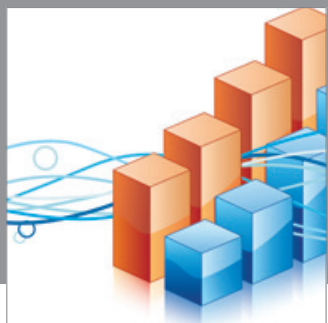

Advances in

Operations Research

mansans

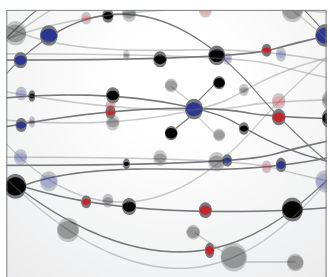

The Scientific World Journal
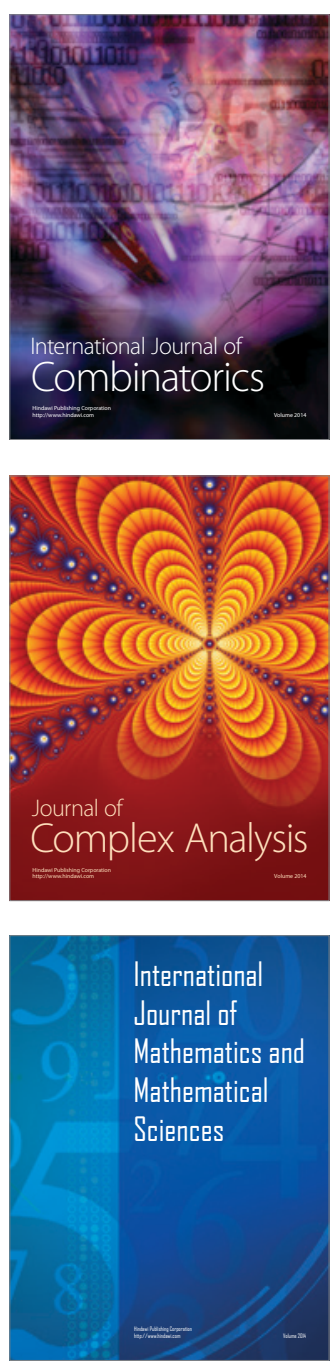
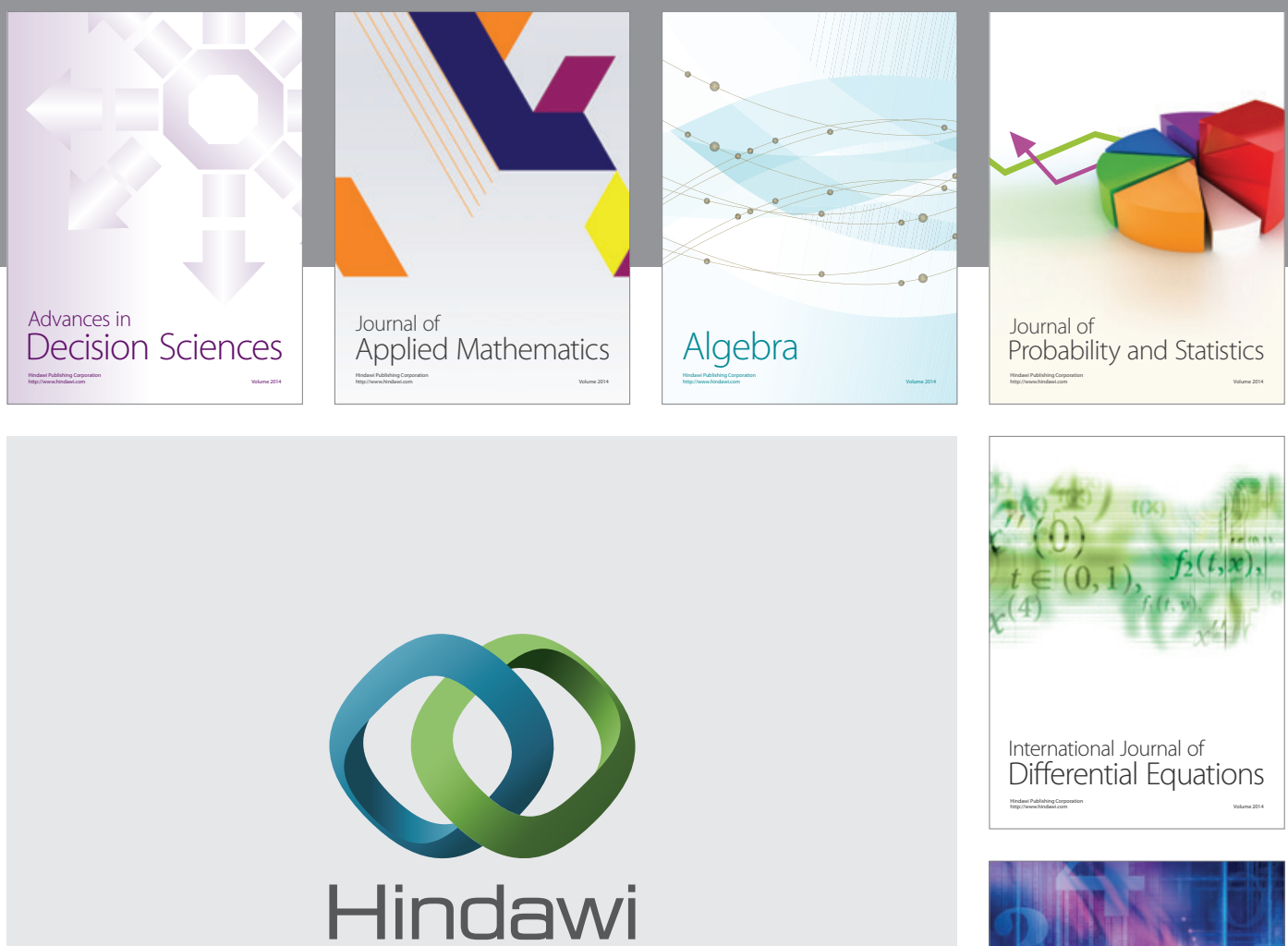

Submit your manuscripts at http://www.hindawi.com
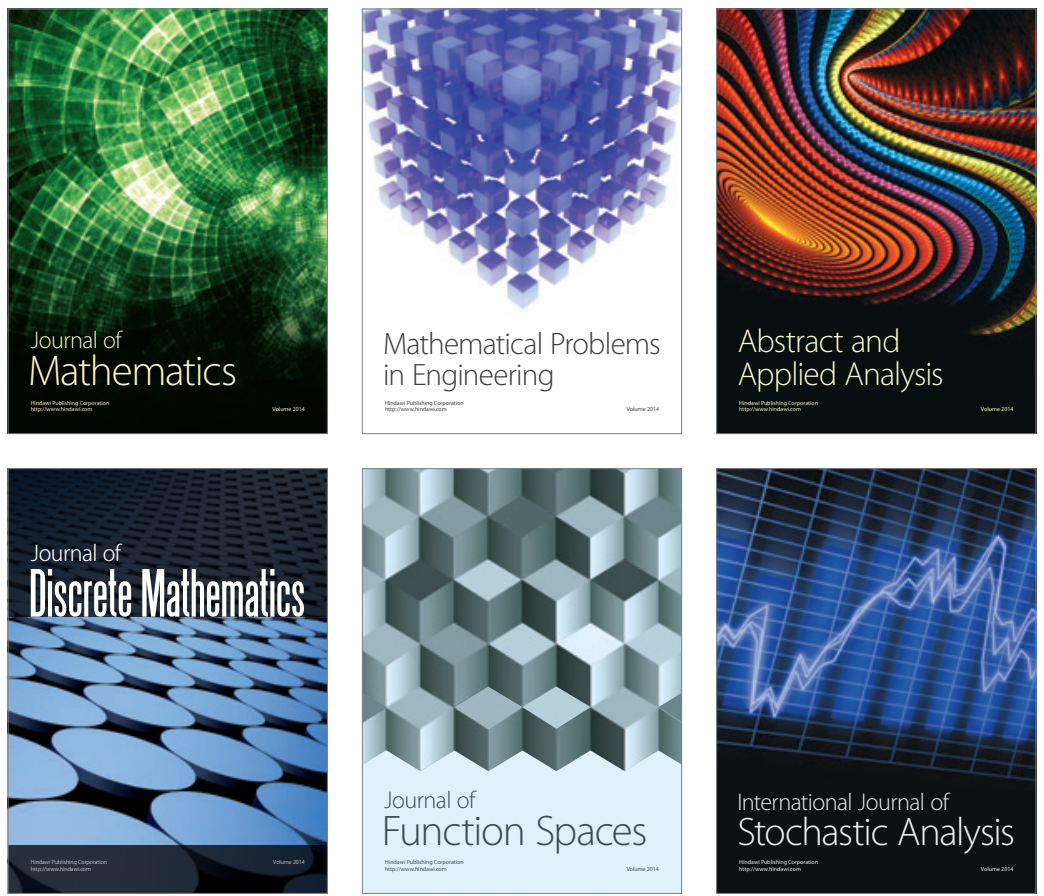

Journal of

Function Spaces

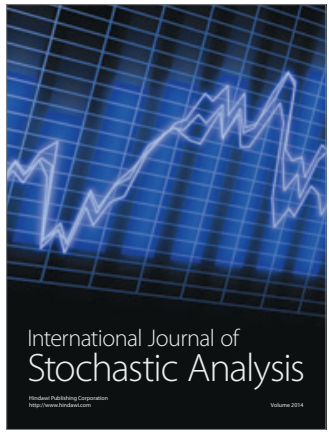

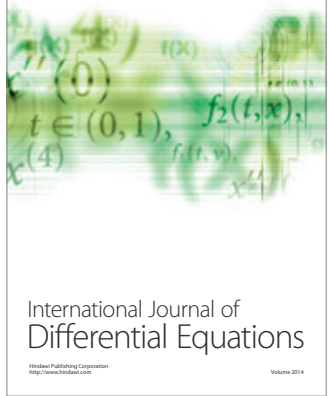
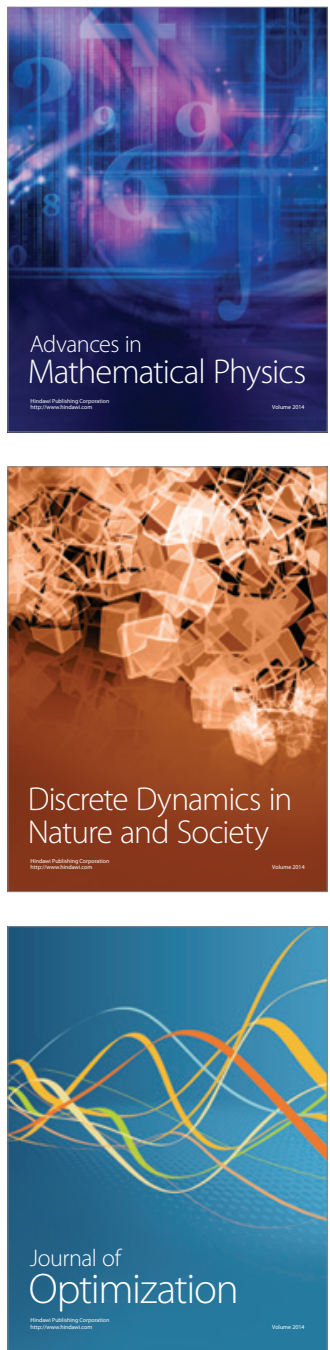